\title{
Uniform Interpolation and Coherence ${ }^{\frac{h_{r}}{r}}$
}

\author{
Tomasz Kowalski \\ Department of Mathematics and Statistics, La Trobe University, Melbourne, Australia \\ George Metcalfe \\ Mathematical Institute, University of Bern, Switzerland
}

\begin{abstract}
A variety $\mathcal{V}$ is said to be coherent if every finitely generated subalgebra of a finitely presented member of $\mathcal{V}$ is finitely presented. It is shown here that coherence corresponds to a key ingredient of uniform deductive interpolation for equational consequence in $\mathcal{V}$ : the property that any compact congruence on a finitely generated free algebra of $\mathcal{V}$ restricted to a free algebra over fewer generators is compact. A general criterion is derived for establishing failures of coherence, and hence also of uniform deductive interpolation. The criterion is then applied in conjunction with properties of canonical extensions to prove that coherence and uniform deductive interpolation fail for certain varieties of Boolean algebras with operators (including varieties for the modal logic $\mathrm{K}$ and KT), double-Heyting algebras, residuated lattices, and lattices.

Keywords: Uniform Interpolation, Coherence, Compact Congruences, Free Algebras, Canonical Extensions, Modal Logics, Residuated Lattices
\end{abstract}

\section{Introduction}

Uniform interpolation was established for intuitionistic propositional logic IPC by Pitts in [37] and used by Ghilardi and Zawadowski in [21] to prove

\footnotetext{
The second author acknowledges support from Swiss National Science Foundation grant 200021_165850 and the European Union's Horizon 2020 research and innovation programme under the Marie Skłodowska-Curie grant agreement No 689176.

Email addresses: T.Kowalski@latrobe.edu.au (Tomasz Kowalski), george.metcalfe@math.unibe.ch (George Metcalfe)
} 
that the first-order theory of Heyting algebras has a model completion. More generally, Ghilardi and Zawadowski proved in [16] that a model completion exists for the first-order theory of any variety (equational class) $\mathcal{V}$ satisfying certain category-theoretic conditions. These conditions were reformulated by van Gool et al. in [17] as properties of equational consequence in $\mathcal{V}$ - most prominently, right and left uniform deductive interpolation - and related to properties of compact congruences on its free and finitely presented algebras. In particular, if $\mathcal{V}$ admits deductive interpolation (or has the amalgamation property), then right uniform deductive interpolation is equivalent to the condition that any compact congruence on a finitely generated free algebra of $\mathcal{V}$ restricted to a free algebra over fewer generators is compact.

In Section 2 of this paper, we prove that the latter condition is equivalent to the property of coherence: namely, any finitely generated subalgebra of a finitely presented member of $\mathcal{V}$ is finitely presented. ${ }^{1}$ This notion of coherence originated in sheaf theory and has been studied quite widely in algebra, mostly in connection with groups, rings, modules, monoids, and lattices (see, e.g., $[4,9,18,38])$. It has also been considered from a more general modeltheoretic perspective by Wheeler $[43,44]$, who proved that coherence of a variety is implied by, and in conjunction with amalgamation and a further property implies, the existence of a model completion for its first-order theory.

Following Pitts' seminal result for IPC [37], uniform interpolation or its failure has been established for a wide range of other non-classical logics. In particular, all eight intermediate logics with Craig interpolation have uniform interpolation [16], but some modal logics with Craig interpolation, including S4 and K4 [3,20], do not. Semantic proofs of uniform interpolation using bisimulation quantifiers were given by Visser in [42] for the modal logic K (proved independently by Ghilardi [15]), Grzegorczyk logic S4Grz, and GödelLöb logic GL (first proved by Shavrukov [39]), and syntactic Pitts-style proofs were given by Bílková in [3] for K and KT. Relationships between uniform interpolation and bisimulation quantifiers for the modal $\mu$-calculus and other fixpoint modal logics have also been studied in some depth in $[7,8,33]$.

Crucially for the topic of this paper, however, the above-mentioned proofs establish an "implication-based" uniform interpolation property that implies the "consequence-based" uniform deductive interpolation property studied

\footnotetext{
${ }^{1}$ Note that the notion of coherence defined and studied by Taylor in [41] is entirely different, and not related to our results.
} 
in [17] for IPC, GL, and S4Grz, but not for K or KT. The same observation applies to uniform interpolation results for substructural logics (or varieties of residuated lattices) obtained by Alizadeh et al. in [1]; conversely, Łukasiewicz logic (or the variety of MV-algebras) has uniform deductive interpolation, but not Craig interpolation (see [17]). In the case of $\mathrm{K}$, the failure of uniform deductive interpolation was already observed (at least implicitly) in [19], where it was shown that the description logic ALC, a notational variant of multi-modal K, does not have this property; moreover, a characterization was given in [30] of the formulas of ALC that have deductive uniform interpolants.

In Section 3, we provide a general criterion for establishing failures of coherence, and hence also of uniform deductive interpolation. The criterion states, roughly, that in a coherent variety $\mathcal{V}$ of algebras with a term-definable semilattice reduct, any decreasing and monotone term that satisfies a fixpoint embedding condition in $\mathcal{V}$ admits a fixpoint obtained by iterating the term finitely many times. In Section 4, we briefly review the theory of canonical extensions and prove two fixpoint lemmas that are then used in Section 5 to obtain a condition for the failure of coherence for varieties of ordered algebras closed under canonical completions. We apply this condition to show that any coherent variety of Boolean algebras with operators that is closed under canonical extensions has equationally definable principal congruences (EDPC). In particular, $\mathrm{K}$ is not coherent, does not admit uniform deductive interpolation, and its first-order theory does not have a model completion. Moreover, the same is true of any normal modal logic closed under canonical extensions for which $\square^{n} x \approx \square^{n+1} x$ fails for all $n \in \mathbb{N}$ (where $\square x:=\square x \wedge x$ ). We obtain similar results also for varieties of residuated lattices, doubleHeyting algebras, and lattices. In the latter case, we obtain an alternative proof of Schmidt's result that the variety of lattices is not coherent and its first-order theory does not have a model completion [38].

Let us note finally that in [28] we have also used the criterion obtained in Section 3 to establish failures of coherence, and hence uniform interpolation, for families of transitive modal logics such as K4 and S4. These results, which overlap with results obtained by Ghilardi and Zawadowski in [16,20], will be discussed briefly in Section 5, referring to [28] for further details and proofs.

\section{Uniform Deductive Interpolation and Coherence}

In this section, we first recall the definitions of deductive interpolation and uniform deductive interpolation for equational consequence in a variety 
and their algebraic characterizations in terms of congruences on free algebras. We then recall the notion of coherence for a variety and relate this notion to uniform deductive interpolation (Theorem 2.3).

Let us assume that $\mathcal{L}$ is an algebraic signature with at least one constant symbol and that $\mathcal{V}$ is a variety of $\mathcal{L}$-algebras. The assumption that $\mathcal{L}$ contains a constant is not essential - indeed it will be dropped when considering varieties of lattices in Subsection 5.4 - but is adopted here for convenience of presentation and easier reference to $[17,32]$.

For any (possibly infinite) set of variables $\bar{x}$, we denote by $\operatorname{Tm}(\bar{x})$ the $\mathcal{L}$-term algebra over $\bar{x}$ and by $\mathbf{F}(\bar{x})$, the free algebra of $\mathcal{V}$ over $\bar{x}$. We write $t(\bar{x}), \varepsilon(\bar{x})$, or $\Sigma(\bar{x})$ to denote that the variables of, respectively, an $\mathcal{L}$-term $t$, $\mathcal{L}$-equation $\varepsilon$, or set of $\mathcal{L}$-equations $\Sigma$ are included in $\bar{x}$. Where appropriate, we deliberately confuse these expressions with the corresponding elements, pairs of elements, and sets of pairs of elements from $\mathbf{F}(\bar{x})$. We also adopt the convention that $\bar{x}, \bar{y}$, etc. denote disjoint sets, and let $\bar{x}, \bar{y}$ denote their disjoint union.

For a set of $\mathcal{L}$-equations $\Sigma \cup\{\varepsilon\}$ containing exactly the variables in the set $\bar{x}$, we define

$$
\begin{gathered}
\Sigma \models_{\mathcal{V}} \varepsilon: \Longleftrightarrow \text { for every } \mathbf{A} \in \mathcal{V} \text { and homomorphism } e: \operatorname{Tm}(\bar{x}) \rightarrow \mathbf{A}, \\
\Sigma \subseteq \operatorname{ker}(e) \Longrightarrow \varepsilon \in \operatorname{ker}(e) .
\end{gathered}
$$

For a set of $\mathcal{L}$-equations $\Sigma \cup \Delta$, we write $\Sigma \models_{\mathcal{V}} \Delta$ if $\Sigma \models_{\mathcal{V}} \varepsilon$ for all $\varepsilon \in \Delta$.

We say that $\mathcal{V}$ admits deductive interpolation if for any sets $\bar{x}, \bar{y}, \bar{z}$ and set of equations $\Sigma(\bar{x}, \bar{y}) \cup\{\varepsilon(\bar{y}, \bar{z})\}$ satisfying $\Sigma \models_{\mathcal{V}} \varepsilon$, there exists a set of equations $\Pi(\bar{y})$ such that $\Sigma \models_{\mathcal{V}} \Pi$ and $\Pi \models \mathcal{V} \varepsilon$. This property has been studied in depth by many authors (see, e.g., [5, 6, 23,31,32,35,36,45]). In particular, it is known that if $\mathcal{V}$ has the amalgamation property, then it admits deductive interpolation, and, conversely, if $\mathcal{V}$ admits deductive interpolation and has the congruence extension property, then it has the amalgamation property (see [32] for proofs and further references). Let us just also note here (see [17] for a proof) that $\mathcal{V}$ admits deductive interpolation if and only if for any finite sets $\bar{x}, \bar{y}$ and finite set of equations $\Sigma(\bar{x}, \bar{y})$, there exists a set of equations $\Pi(\bar{y})$ such that for any equation $\varepsilon(\bar{y}, \bar{z})$,

$$
\Sigma \models_{\mathcal{V}} \varepsilon \Longleftrightarrow \Pi \models_{\mathcal{V}} \varepsilon \text {. }
$$

Following [17], we say that $\mathcal{V}$ admits right uniform deductive interpolation if $\Pi(\bar{y})$ in the preceding condition is required to be finite. 
To reformulate these notions via congruences on free algebras of $\mathcal{V}$, let us denote the congruence on an algebra $\mathbf{A}$ generated by $S \subseteq A^{2}$ by $\operatorname{Cg}_{\mathbf{A}}(S)$, and recall (see [32, Lemma 2]) that for any sets of equations $\Sigma(\bar{x}), \Delta(\bar{x})$,

$$
\Sigma \models \mathcal{V} \Delta \Longleftrightarrow \operatorname{Cg}_{\mathbf{F}(\bar{x})}(\Delta) \subseteq \operatorname{Cg}_{\mathbf{F}(\bar{x})}(\Sigma)
$$

Let us also denote the congruence lattice of an algebra $\mathbf{A}$ by $\mathrm{Con} \mathbf{A}$, and recall that the adjoint lifting of a homomorphism $h: \mathbf{A} \rightarrow \mathbf{B}$ in $\mathcal{V}$ to the congruence lattices of $\mathbf{A}$ and $\mathbf{B}$ is the adjoint pair of maps

$$
\begin{gathered}
h^{*}: \text { Con } \mathbf{A} \leftrightarrows \operatorname{Con} \mathbf{B}: h^{-1}, \\
h^{*}(\Psi):=\operatorname{Cg}_{\mathbf{B}}\left(\left\{\left\langle h(a), h\left(a^{\prime}\right)\right\rangle \mid\left\langle a, a^{\prime}\right\rangle \in \Psi\right\}\right), \\
h^{-1}(\Theta):=\left\{\left\langle a, a^{\prime}\right\rangle \in A^{2} \mid\left\langle h(a), h\left(a^{\prime}\right)\right\rangle \in \Theta\right\}=\operatorname{ker}(h)(-) / \Theta .
\end{gathered}
$$

It is easily checked that $\mathcal{V}$ admits deductive interpolation if and only if for any finite sets $\bar{x}, \bar{y}, \bar{z}$, the following diagram commutes:

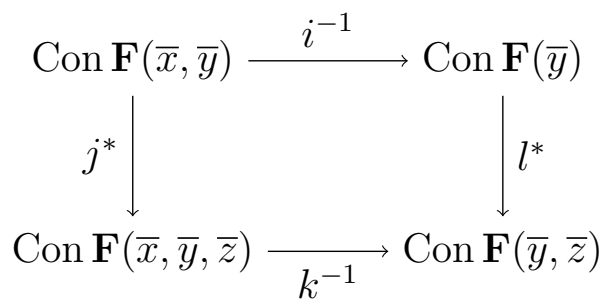

where $i, j, k$, and $l$ are the inclusion maps between corresponding finitely generated free algebras.

Let us denote the set of compact (finitely generated) congruences on an algebra $\mathbf{A}$ by KCon $\mathbf{A}$, noting that KCon $\mathbf{A}$ is always a join-subsemilattice of Con $\mathbf{A}$, but meets in KCon $\mathbf{A}$ need not exist in general. For a homomorphism $h: \mathbf{A} \rightarrow \mathbf{B}$, the map $h^{*}$ restricts to a map $\left.h^{*}\right|_{\text {KCon } \mathbf{A}}:$ KCon $\mathbf{A} \rightarrow$ KCon $\mathbf{B}$, which we call the compact lifting of $h$. On the other hand, $h^{-1}$ restricts to $\left.h^{-1}\right|_{\mathrm{KCon} \mathbf{B}}: \mathrm{KCon} \mathbf{B} \rightarrow \mathrm{KCon} \mathbf{A}$, the right adjoint of $\left.h^{*}\right|_{\mathrm{KCon} \mathbf{A}}$, if and only if $h$ preserves compact congruences. The existence of right adjoints for compact liftings between free and finitely presented algebras is related to a weaker form of right uniform deductive interpolation where the considered equations $\varepsilon$ contain only variables in $\bar{y}$.

Proposition 2.1 (cf. [17, Proposition 3.8]). The following are equivalent: 
(1) For any finite sets $\bar{x}, \bar{y}$ and finite set of equations $\Sigma(\bar{x}, \bar{y})$, there exists a finite set of equations $\Pi(\bar{y})$ such that for any equation $\varepsilon(\bar{y})$,

$$
\Sigma \models_{\mathcal{V}} \varepsilon \Longleftrightarrow \Pi \models_{\mathcal{V}} \varepsilon
$$

(2) For any finite sets $\bar{x}, \bar{y}$ and compact congruence $\Theta$ on $\mathbf{F}(\bar{x}, \bar{y})$, the congruence $\Theta \cap F(\bar{y})^{2}$ on $\mathbf{F}(\bar{y})$ is compact.

(3) For any finite sets $\bar{x}, \bar{y}$, the compact lifting of the inclusion map from $\mathbf{F}(\bar{y})$ to $\mathbf{F}(\bar{x}, \bar{y})$ has a right adjoint.

(4) The compact lifting of any homomorphism between finitely presented algebras in $\mathcal{V}$ has a right adjoint.

We will show that the conditions of this proposition are equivalent also to a further well-studied property from algebra, but first we establish a useful technical result, proved in a slightly different form as Lemma 3.9 in [17].

Lemma 2.2. Suppose that $f: \mathbf{F}(\bar{y}) \rightarrow \mathbf{A}$ and $g: \mathbf{F}(\bar{x}) \rightarrow \mathbf{A}$ are surjective homomorphisms in $\mathcal{V}$ and let $r: \mathbf{F}(\bar{y}) \rightarrow \mathbf{F}(\bar{x})$ and $s: \mathbf{F}(\bar{x}) \rightarrow \mathbf{F}(\bar{y})$ be the natural maps satisfying $f=g \circ r$ and $g=f \circ s$. If $\operatorname{ker}(g)$ is generated by $\Pi \subseteq F(\bar{x})^{2}$, then $\operatorname{ker}(f)$ is generated by $\Sigma=\{\langle s(a), s(b)\rangle \mid\langle a, b\rangle \in$ $\Pi\} \cup\{\langle y, \operatorname{sr}(y)\rangle \mid y \in \bar{y}\} \subseteq F\left(\bar{y}^{2}\right)$.

Proof. The situation is depicted in the following diagram:

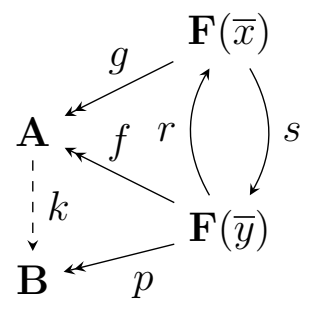

Observe first that $\Sigma \subseteq \operatorname{ker}(f)$. For any $\langle a, b\rangle \in \Pi$, we have $g(a)=g(b)$ and so $f s(a)=g(a)=g(b)=f s(b)$; that is, $\langle s(a), s(b)\rangle \in \operatorname{ker}(f)$. Also, given any $y \in \bar{y}$, we have $f(y)=g r(y)=f \operatorname{sr}(y)$; that is, $\langle y, \operatorname{sr}(y)\rangle \in \operatorname{ker}(f)$.

Now let $\Psi=\operatorname{Cg}_{\mathbf{F}(\bar{y})}(\Sigma)$. Define $\mathbf{B}=\mathbf{F}(\bar{y}) / \Psi$ and let $p: \mathbf{F}(\bar{y}) \rightarrow \mathbf{B}$ be the natural homomorphism with $\Psi=\operatorname{ker}(p) \subseteq \operatorname{ker}(f)$. Let $h=p \circ s$, and observe that for $\langle a, b\rangle \in \Pi$, we have $h(a)=p s(a)=p s(b)=h(b)$, since $\langle s(a), s(b)\rangle \in \Psi$. This implies $\operatorname{ker}(g) \subseteq \operatorname{ker}(h)$, and hence there exists a unique homomorphism $k: \mathbf{A} \rightarrow \mathbf{B}$ such that $k \circ g=h$. For each $y \in \bar{y}$,

$$
p(y)=\operatorname{psr}(y)=h r(y)=\operatorname{kgr}(y)=k f(y),
$$


which, by freeness, implies that $p=k f$. But then $\Psi=\operatorname{ker}(p) \supseteq \operatorname{ker}(f)$, yielding $\Psi=\operatorname{ker}(f)$ as required.

Note that if $\Pi$ and $\bar{y}$ in Lemma 2.2 are finite, then $\Sigma$ is also finite. Other properties of $\Pi$, such as being recursive, also transfer to $\Sigma$ under certain further mild assumptions, but this will not concern us here.

Following Wheeler [43], let us call $\mathcal{V}$ coherent if every finitely generated subalgebra of a finitely presented member of $\mathcal{V}$ is finitely presented. It is proved in [43] that the coherence of $\mathcal{V}$ is implied by, and in conjunction with amalgamation and one further property implies, the existence of a model completion for the first-order theory of $\mathcal{V}$.

We now prove the main result of this section.

Theorem 2.3. $\mathcal{V}$ is coherent if and only if any of the equivalent conditions of Proposition 2.1 holds.

Proof. Assume that $\mathcal{V}$ is coherent. We will prove that condition (2) of Proposition 2.1 holds. Let $\Theta$ be a compact congruence on $\mathbf{F}(\bar{y}, \bar{z})$, so that $\mathbf{C}=\mathbf{F}(\bar{y}, \bar{z}) / \Theta$ is finitely presented. Let $\Psi=\Theta \cap F(\bar{y})^{2}$ and $\mathbf{A}=\mathbf{F}(\bar{y}) / \Psi$. Then $\mathbf{A}$ is finitely generated and embeds into $\mathbf{C}$. By coherence, $\mathbf{A}$ is finitely presented. Hence let $\mathbf{F}(\bar{x}) / \Phi \cong \mathbf{A}$ be a finite presentation such that $\Pi$ is a finite set of generators of $\Phi$. Let $f: \mathbf{F}(\bar{y}) \rightarrow \mathbf{A}$ and $g: \mathbf{F}(\bar{x}) \rightarrow \mathbf{A}$ be the surjective homomorphisms such that $\Psi=\operatorname{ker}(f)$ and $\Phi=\operatorname{ker}(g)$, and let $r: \mathbf{F}(\bar{y}) \rightarrow \mathbf{F}(\bar{x})$ and $s: \mathbf{F}(\bar{x}) \rightarrow \mathbf{F}(\bar{y})$ be the natural maps satisfying $f=g \circ r$ and $g=f \circ s$. Then the assumptions of Lemma 2.2 are satisfied, so $\Psi$ is generated by a finite set. That is, $\Psi$ is compact, as required.

Assume now that condition (2) of Proposition 2.1 holds. Let B be a finitely generated subalgebra of a finitely presented algebra $\mathbf{A} \in \mathcal{V}$, and let $\bar{x}, \bar{y}$ and $\bar{y}$ be finite sets generating $\mathbf{A}$ and $\mathbf{B}$, respectively. The surjective homomorphism $h: \mathbf{F}(\bar{x}, \bar{y}) \rightarrow \mathbf{A}$ extending the identity map on $\bar{x}, \bar{y}$ restricts to $k: \mathbf{F}(\bar{y}) \rightarrow \mathbf{B}$, which is also surjective. But $\operatorname{ker}(h) \in \operatorname{KCon} \mathbf{F}(\bar{x}, \bar{y})$ by Lemma 2.2, so by assumption, $\operatorname{ker}(k)=\operatorname{ker}(h) \cap F(\bar{y})^{2} \in \operatorname{KCon} \mathbf{F}(\bar{y})$. Hence $\mathbf{B}$ is finitely presented.

Combining Theorem 2.3 with [17, Proposition 3.5], we obtain also the following characterization of right uniform deductive interpolation.

Proposition 2.4. $\mathcal{V}$ admits right uniform deductive interpolation if and only if $\mathcal{V}$ is coherent and admits deductive interpolation. 
A similar characterization has been obtained for a left uniform deductive interpolation property (see [17, Proposition 4.3]). However, in this paper, we focus only on failures of right uniform deductive interpolation, indeed only on cases where coherence fails.

Example 2.5. Clearly, every locally finite variety is coherent. Less obviously, the property holds for the varieties of Heyting algebras (the main content of Pitts' theorem for IPC [37]), abelian groups, lattice-ordered abelian groups, and MV-algebras (see [17]). On the other hand, the variety of groups is not coherent, since there exists a finitely generated recursively presented group that is not finitely presented, but, by Higman's embedding theorem (cf. [22]), embeds into a finitely presented group.

The reasoning outlined in Example 2.5 for the variety of groups can be generalized to produce further failures of coherence (e.g., for the variety of monoids). Following [25], let us say that $\mathcal{V}$ has the Higman property if every finitely generated recursively presented algebra in $\mathcal{V}$ embeds into a finitely presented algebra in $\mathcal{V}$.

Proposition 2.6. If every finitely generated recursively presented algebra in $\mathcal{V}$ is finitely presented, then $\mathcal{V}$ is coherent. Moreover, if $\mathcal{V}$ satisfies the Higman property, then the converse also holds.

Proof. First we prove that a certain converse to the Higman property holds: namely, if $\mathbf{A}$ is a finitely generated subalgebra of some finitely presented $\mathbf{B} \in \mathcal{V}$, then $\mathbf{A}$ is recursively presented. As in the proof of Theorem 2.3, we may assume without loss of generality that the set of generators of $\mathbf{A}$ is contained in the set of generators of $\mathbf{B}$. Suppose then that $\mathbf{B} \cong \mathbf{F}(\bar{x}, \bar{y}) / \Theta$ for some compact congruence $\Theta$ and $\mathbf{A} \cong \mathbf{F}(\bar{x}) / \Psi$, where $\Psi=\Theta \cap \mathbf{F}(\bar{x})^{2}$. Since $\Theta$ is compact and $\bar{x}$ is finite, $\Psi$ is recursively generated. Hence $\mathbf{A}$ is recursively presented as claimed.

Now assume that every finitely generated recursively presented algebra in $\mathcal{V}$ is finitely presented, and consider a finitely generated subalgebra $\mathbf{A}$ of some finitely presented $\mathbf{B} \in \mathcal{V}$. As we have just shown, $\mathbf{A}$ must be recursively presented, and hence $\mathbf{A}$ is finitely presented. The remaining part is clear.

\section{A General Criterion}

The main result of this section, Theorem 3.1, establishes that in any coherent variety $\mathcal{V}$ of algebras with a term-definable meet-semilattice reduct, 
each term $t(x, \bar{u})$ that is decreasing and monotone, and satisfies a fixpoint embedding condition (with respect to $x$ ), has a fixpoint obtained by iterating $t$ finitely many times in the first argument. Understood contrapositively, this result provides a general criterion for establishing the failure of coherence and therefore also the failure of right uniform deductive interpolation and the lack of a model completion for the first-order theory of $\mathcal{V}$.

Given any term $t(x, \bar{u})$, we define inductively

$$
t^{0}(x, \bar{u}):=x \quad \text { and } \quad t^{k+1}(x, \bar{u}):=t\left(t^{k}(x, \bar{u}), \bar{u}\right) \text { for } k \in \mathbb{N} .
$$

Let $n \in \mathbb{N}$. We say that $t$ is $n$-potent (with respect to $x$ ) in $\mathcal{V}$ if

$$
\mathcal{V} \models t^{n+1}(x, \bar{u}) \approx t^{n}(x, \bar{u}) .
$$

For any algebra $\mathbf{A}$ and term $s\left(x_{1}, \ldots, x_{n}\right)$, the term function $s^{\mathbf{A}}: A^{n} \rightarrow A$ is defined inductively in the usual way; for convenience, however, we often omit the superscript ${ }^{\mathbf{A}}$ when referring to such functions.

Theorem 3.1. Let $\mathcal{V}$ be a coherent variety of $\mathcal{L}$-algebras with a termdefinable meet-semilattice reduct and a term $t(x, \bar{u})$ satisfying

$$
\mathcal{V}=t(x, \bar{u}) \leq x \quad \text { and } \quad \mathcal{V} \models x \leq y \Rightarrow t(x, \bar{u}) \leq t(y, \bar{u})
$$

Suppose also that $\mathcal{V}$ satisfies the following fixpoint embedding condition with respect to $t(x, \bar{u})$ :

(FE) For any finitely generated $\mathbf{A} \in \mathcal{V}$ and $a, \bar{b} \in A$, there exists $\mathbf{B} \in \mathcal{V}$ such that $\mathbf{A}$ is a subalgebra of $\mathbf{B}$ and $\bigwedge_{k \in \mathbb{N}} t^{k}(a, \bar{b})$ exists in $\mathbf{B}$, satisfying

$$
\bigwedge_{k \in \mathbb{N}} t^{k}(a, \bar{b})=t\left(\bigwedge_{k \in \mathbb{N}} t^{k}(a, \bar{b}), \bar{b}\right)
$$

Then $t$ is $n$-potent (with respect to $x$ ) in $\mathcal{V}$ for some $n \in \mathbb{N}$.

Proof. Let $\mathcal{V}$ and $t(x, \bar{u})$ be as in the statement of the theorem. Note first that the fact that $t$ is decreasing and monotone easily implies that for any $n \in \mathbb{N}$,

$$
\mathcal{V} \models t^{n+1}(x, \bar{u}) \leq t^{n}(x, \bar{u}) .
$$

To establish the converse inequality for some $n \in \mathbb{N}$, we define

$$
\Sigma=\{y \leq x, x \leq z, x \approx t(x, \bar{u})\} \quad \text { and } \quad \Pi=\left\{y \leq t^{k}(z, \bar{u}) \mid k \in \mathbb{N}\right\}
$$


and prove that for any equation $\varepsilon(y, z, \bar{u})$,

$$
\Sigma \models_{\mathcal{V}} \varepsilon(y, z, \bar{u}) \Longleftrightarrow \Pi \models_{\mathcal{V}} \varepsilon(y, z, \bar{u}) .
$$

For the right-to-left direction, it suffices to observe that $\Sigma \models \mathcal{v} y \leq t^{k}(z, \bar{u})$ for each $k \in \mathbb{N}$. For the left-to-right direction, suppose contrapositively that $\Pi \not \forall_{\mathcal{V}} \varepsilon(y, z, \bar{u})$. Since only finitely many variables occur in $\Pi$, there exist a finitely generated $\mathbf{A} \in \mathcal{V}$ and a homomorphism $e: \operatorname{Tm}(y, z, \bar{u}) \rightarrow \mathbf{A}$ such that $\Pi \subseteq \operatorname{ker}(e)$, but $\varepsilon \notin \operatorname{ker}(e)$. Let $a=e(y), b=e(z)$, and $\bar{c}=e(\bar{u})$. By assumption, $\mathbf{A}$ is a subalgebra of some $\mathbf{B} \in \mathcal{V}$ such that $\bigwedge_{k \in \mathbb{N}} t^{k}(b, \bar{c})$ exists in $\mathbf{B}$ and

$$
\bigwedge_{k \in \mathbb{N}} t^{k}(b, \bar{c})=t\left(\bigwedge_{k \in \mathbb{N}} t^{k}(b, \bar{c}), \bar{c}\right) .
$$

Since $x$ does not appear in $\Pi \cup\{\varepsilon\}$, we may extend $e$ to a homomorphism $e: \operatorname{Tm}(x, y, z, \bar{u}) \rightarrow \mathbf{B}$ by defining

$$
e(x)=\bigwedge_{k \in \mathbb{N}} t^{k}(b, \bar{c})
$$

But $a \leq t^{k}(b, \bar{c}) \leq b$ for each $k \in \mathbb{N}$, so clearly $a \leq e(x) \leq b$. Moreover, by the fixpoint embedding condition,

$$
e(x)=\bigwedge_{k \in \mathbb{N}} t^{k}(b, \bar{c})=t\left(\bigwedge_{k \in \mathbb{N}} t^{k}(b, \bar{c}), \bar{c}\right)=e(t(x, \bar{u}))
$$

Hence $\Sigma \subseteq \operatorname{ker}(e)$ and we obtain $\Sigma \not \not_{\mathcal{V}} \varepsilon(y, z, \bar{u})$, completing the proof of $(\star)$.

Since $\mathcal{V}$ is coherent, there exists a finite set of equations $\Delta(y, z, \bar{u})$ such that for any equation $\varepsilon(y, z, \bar{u})$,

$$
\Sigma \models_{\mathcal{V}} \varepsilon(y, z, \bar{u}) \Longleftrightarrow \Delta \models_{\mathcal{V}} \varepsilon(y, z, \bar{u}) .
$$

So $\Sigma \models \mathcal{V} \Delta$, and, by $(\star)$, also $\Pi \models \mathcal{V} \Delta$. Using the compactness of $\models_{\mathcal{V}}$ (see [32]) and the fact that $\Delta$ is finite, $\Pi^{\prime} \models \mathcal{V} \Delta$ for some finite $\Pi^{\prime} \subseteq \Pi$. But also $\left\{y \leq t^{k+1}(z, \bar{u})\right\} \models \mathcal{V} y \leq t^{k}(z, \bar{u})$ for each $k \in \mathbb{N}$, and hence for some $n \in \mathbb{N}$,

$$
\left\{y \leq t^{n}(z, \bar{u})\right\} \models \mathcal{v} \Delta .
$$

Since $\Sigma \models_{\mathcal{V}} y \leq t^{n+1}(z, \bar{u})$, also $\Delta \models_{\mathcal{V}} y \leq t^{n+1}(z, \bar{u})$. Hence, combining these consequences, $\left\{y \leq t^{n}(z, \bar{u})\right\} \models \mathcal{V} y \leq t^{n+1}(z, \bar{u})$. Finally, substituting $y$ with $t^{n}(z, \bar{u})$ and $z$ with $x$, we obtain $\mathcal{V} \models t^{n}(x, \bar{u}) \leq t^{n+1}(x, \bar{u})$. 
The proof of Theorem 3.1 can be used to obtain direct counterexamples to coherence. Suppose that $\mathcal{V}$ satisfies the conditions of the theorem and that $t(x, \bar{u})$ is not $n$-potent in $\mathcal{V}$ for some $n \in \mathbb{N}$. Let $\Theta$ be the compact congruence on $\mathbf{F}(x, y, z, \bar{u})$ generated by $\{y \leq x, x \leq z, x \approx t(x)\}$, and let $\Psi$ be the congruence on $\mathbf{F}(y, z, \bar{u})$ generated by $\left\{y \leq t^{n}(z) \mid n \in \mathbb{N}\right\}$. Then $\mathbf{F}(y, z, \bar{u}) / \Psi$ is a finitely generated but not finitely presented member of $\mathcal{V}$ that embeds into the finitely presented algebra $\mathbf{F}(x, y, z, \bar{u}) / \Theta$ of $\mathcal{V}$.

\section{Canonical extensions}

In this section, we describe a second tool for establishing the failure of coherence and uniform deductive interpolation for a variety: the theory of canonical extensions. To keep the paper reasonably self-contained, we begin with a brief review of this theory based on the development in [14], from which we depart only slightly by considering unbounded lattices (see Remark 2.9 of [14] for technicalities). The reader familiar with canonical extensions may skip this section, with the exception of Lemmas 4.9 and 4.10 that are required for the applications in Section 5, and do not appear in [14]; Lemma 4.9 is a reformulation of Esakia's Lemma [10], while Lemma 4.10 seems to be new.

A completion of a lattice $\mathbf{L}$ is a pair $\langle e, \mathbf{C}\rangle$, where $\mathbf{C}$ is a complete lattice and $e$ is an order embedding of $\mathbf{L}$ into $\mathbf{C}$ that preserves all existing finite meets and joins of $\mathbf{L}$. An element $a \in C$ is called open if $a=\bigvee e(X)$ for some subset $X$ of $L$, where the join is taken in $C$; note that in this case $X$ can be taken to be the set $\{x \in L \mid a \leq e(x)\}$. Dually, $a \in C$ is closed if $a=\bigwedge e(X)$ for some $X \subseteq L$. We will use $K$ and $O$ to denote the sets of closed and open elements of $\mathbf{C}$, respectively. A completion $\langle e, \mathbf{C}\rangle$ is called

- dense if every element of $C$ is both a join of closed elements and a meet of open elements;

- compact if for any $A \subseteq K$ and $B \subseteq O$, we have $\bigwedge A \leq \bigvee B$ if and only if there are finite subsets $A_{0}$ of $A$ and $B_{0}$ of $B$ satisfying $\wedge A_{0} \leq \bigvee B_{0}$.

A dense and compact completion $\langle e, \mathbf{C}\rangle$ of $\mathbf{L}$ is called a canonical extension.

Theorem 4.1. Any lattice $\mathbf{L}$ has a canonical extension $\langle e, \mathbf{C}\rangle$. Moreover, if $\left\langle e^{\prime}, \mathbf{C}^{\prime}\right\rangle$ is another canonical extension of $\mathbf{L}$, then there exists a lattice isomorphism $i: \mathbf{C}^{\prime} \rightarrow \mathbf{C}$ such that $i \circ e^{\prime}=e$.

Following standard practice, from now on we will speak of the canonical extension of $\mathbf{L}$, denoted by $\mathbf{L}^{\sigma}$. We will also assume that the embedding is realised as the natural identity embedding, so that $\mathbf{L}$ is a sublattice of $\mathbf{L}^{\sigma}$. 
Maps between lattices also have canonical extensions. Let $\mathbf{L}$ and $\mathbf{M}$ be lattices, and let $f: L \rightarrow M$ be any map. The maps $f^{\sigma}, f^{\pi}: L^{\sigma} \rightarrow M^{\sigma}$ are defined as follows:

$$
\begin{aligned}
& f^{\sigma}(x)=\bigvee\{\bigwedge\{f(a) \mid a \in L, p \leq a \leq q\} \mid p \in K, q \in O, p \leq x \leq q\} \\
& f^{\pi}(x)=\bigwedge\{\bigvee\{f(a) \mid a \in L, p \leq a \leq q\} \mid p \in K, q \in O, p \leq x \leq q\}
\end{aligned}
$$

The following two lemmas are easy consequences of these definitions.

Lemma 4.2. Both $f^{\sigma}$ and $f^{\pi}$ extend $f$. Moreover, $f^{\sigma} \leq f^{\pi}$ under the pointwise ordering.

Lemma 4.3. Let $f: L \rightarrow M$ be an order-preserving map.

(a) $f^{\sigma}(p)=\bigwedge\{f(a) \mid a \in L, p \leq a\}$, for all $p \in K$;

(b) $f^{\pi}(q)=\bigvee\{f(a) \mid a \in L, q \geq a\}$, for all $q \in O$;

(c) $f^{\sigma}(x)=\bigvee\left\{f^{\sigma}(p) \mid p \in K, p \leq x\right\}$, for all $x \in L^{\sigma}$;

(d) $f^{\pi}(x)=\bigwedge\left\{f^{\pi}(q) \mid q \in O, q \geq x\right\}$, for all $x \in L^{\sigma}$;

(e) $f^{\sigma}$ and $f^{\pi}$ are equal on $K \cup O$.

If $f^{\sigma}=f^{\pi}$, then we say that $f$ is smooth. An example of a non-smooth map is the implication on a Heyting algebra, viewed as a binary map from $A^{\partial} \times A$ to $A$. The same holds for the residuals of any order-preserving multiplication, so definitions of canonical extensions of residuated structures (see Section 5) must take this into account. To be more precise,

$$
y \leq^{\sigma} x \backslash^{\pi} z \Longleftrightarrow x \cdot^{\sigma} y \leq^{\sigma} z \Longleftrightarrow x \leq^{\sigma} z /^{\pi} y
$$

but these equivalences fail for $\backslash^{\sigma}$ and $/{ }^{\sigma}$. This example also illustrates how to obtain canonical extensions of lattices with additional algebraic structure: since an $n$-ary operation $f$ on a lattice $\mathbf{L}$ is a map $f: L^{n} \rightarrow L$, we naturally obtain extensions $f^{\sigma}:\left(L^{n}\right)^{\sigma} \rightarrow L^{\sigma}$ and $f^{\pi}:\left(L^{n}\right)^{\sigma} \rightarrow L^{\sigma}$. The extensions $\sigma$ and $\pi$ commute with homomorphic images, substructures, and finite direct products, so in particular, $\left(\mathbf{L}^{n}\right)^{\sigma}=\left(\mathbf{L}^{\sigma}\right)^{n}$ and $\left(\mathbf{L}^{n}\right)^{\pi}=\left(\mathbf{L}^{\pi}\right)^{n}$ for any $n \in \mathbb{N}$ and hence canonical extensions of operations are computed coordinatewise.

The next lemma summarises the commutability of canonical extensions with some important class operators; see [14] for details, in particular, Lemma 6.7 for Boolean products. 
Lemma 4.4. Let $\mathcal{K}$ be a class of algebras with term-definable lattice reducts. Then canonical extensions (both $\sigma$ and $\pi$ ) of algebras from $\mathcal{K}$ commute with homomorphic images, subalgebras, finite direct products, and arbitrary Boolean products.

If an operation is order-preserving in some coordinates and order-inverting in others, then it is often necessary to mix and match $\mathbf{L}$ with $\mathbf{L}^{\partial}$ accordingly, as shown by the residuation example above. For our purposes, the maps obtained in this way will be all we need. We will call them isotone from now on, and treat them simply as order-preserving in each coordinate, trusting the reader to work out the appropriate dualisations.

Extensions of arbitrary maps do not behave well under composition, but extensions of isotone maps are better behaved.

Lemma 4.5. Let $\mathbf{L}$ be a lattice, and let $f: L^{n} \rightarrow L$ and $g_{1}, \ldots, g_{n}: L^{k} \rightarrow L$ be isotone maps. Then

(a) $\left(f\left(g_{1}, \ldots, g_{n}\right)\right)^{\sigma} \leq f^{\sigma}\left(g_{1}^{\sigma}, \ldots, g_{n}^{\sigma}\right) \leq f^{\sigma}\left(g_{1}^{\pi}, \ldots, g_{n}^{\pi}\right)$;

(b) $f^{\pi}\left(g_{1}^{\sigma}, \ldots, g_{n}^{\sigma}\right) \leq f^{\pi}\left(g_{1}^{\pi}, \ldots, g_{n}^{\pi}\right) \leq\left(f\left(g_{1}, \ldots, g_{n}\right)\right)^{\pi}$.

We will call a class of algebras $\mathcal{C}$ a class of lattice-ordered algebras if the following conditions hold:

(i) Every algebra in $\mathcal{C}$ has a (uniformly) term-definable lattice reduct.

(ii) Each operation $o$ in the signature of $\mathcal{C}$ has a canonical extension, $o^{\sigma}$ or $o^{\pi}$, determined by $\mathcal{C}$.

If $\mathcal{C}=\{\mathbf{A}\}$, then we just call $\mathbf{A}$ a lattice-ordered algebra. For any $\mathbf{A} \in \mathcal{C}$, we let $\mathbf{A}^{\sigma}$ denote the universe of the canonical extension of the lattice reduct of $\mathbf{A}$ equipped with the canonical extensions of the operations of $\mathbf{A}$.

Let $\mathcal{C}$ be a class of lattice-ordered algebras. Following [24], a term $t$ will be called

- $\mathcal{C}$-expanding if $t^{\mathbf{A}^{\sigma}} \geq\left(t^{\mathbf{A}}\right)^{\sigma}$, for all $\mathbf{A} \in \mathcal{C}$;

- $\mathcal{C}$-contracting if $t^{\mathbf{A}^{\sigma}} \leq\left(t^{\mathbf{A}}\right)^{\sigma}$, for all $\mathbf{A} \in \mathcal{C}$;

- $\mathcal{C}$-stable if $t^{\mathbf{A}^{\sigma}}=\left(t^{\mathbf{A}}\right)^{\sigma}$, for all $\mathbf{A} \in \mathcal{C}$.

For a single algebra $\mathbf{A}$, we will write $\mathbf{A}$-expanding (contracting, stable), instead of the formally correct $\{\mathbf{A}\}$-expanding, etc. By analogy, we will use $\mathcal{C}$-isotone and $\mathbf{A}$-isotone to mean, respectively, isotone on each member of $\mathcal{C}$, and isotone on each member of $\{\mathbf{A}\}$, i.e., isotone in $\mathbf{A}$. Note that, despite the analogy, isotonicity is a property a term has with respect to a single class, 
whereas being expanding, contracting, or stable are properties a term has with respect to a pair of classes: the class $\mathcal{C}$ and the class $\mathcal{C}^{\sigma}=\left\{\mathbf{C}^{\sigma} \mid \mathbf{C} \in \mathcal{C}\right\}$. Often, $\mathcal{C}$ will be clear from the context and omitted. These definitions can obviously be extended to cover $\pi$ extensions and mixed cases, but are not needed here.

Not all isotone terms are stable, but several important ones are: e.g., the lattice operations and the Boolean complement. The next lemma makes this observation precise.

Lemma 4.6. For a lattice $\mathbf{L}$, the extensions $\wedge^{\sigma}$ and $\wedge^{\pi}$ are equal to the meet in $\mathbf{L}^{\sigma}$. Similarly, $\vee^{\sigma}$ and $\vee^{\pi}$ are equal to the join in $\mathbf{L}^{\sigma}$. If $\mathbf{L}$ is distributive, then so is $\mathbf{L}^{\sigma}$. If $\mathbf{B}$ is a Boolean algebra, then so is $\mathbf{B}^{\sigma}$; moreover, $\neg^{\sigma}$ and $\neg^{\pi}$ are both equal to the Boolean complement in $\mathbf{B}^{\sigma}$.

Corollary 4.7. For any class $\mathcal{C}$ of lattice-ordered algebras, the set of $\mathcal{C}$ expanding terms is a clone.

Let us also recall some basic facts about operators. A map $f: P^{n} \rightarrow P$ is an operator if it preserves existing finite joins in each coordinate. A map $g$ is a dual operator if it preserves existing finite meets in each coordinate. Recall that these definitions implicitly incorporate appropriate dualisations of coordinates. In particular, the implication of a Heyting algebra $\mathbf{A}$ is a dual operator when considered as a map from $A^{\partial} \times A$ to $A$. Operators are also called additive operators, and dual operators, multiplicative operators, in particular, in the context of Boolean algebras with operators.

Lemma 4.8. Let $\mathbf{L}$ be a lattice and let $f: L^{n} \rightarrow L$ be an operator and $g_{1}, \ldots g_{n}: L^{k} \rightarrow L$ isotone maps. Assume that the dualisations of the coordinates that make $f$ an operator agree with those that make $g_{1}, \ldots g_{n}$ order-preserving. Then

(a) $f^{\sigma}$ preserves arbitrary non-empty joins in each coordinate;

(b) $f^{\sigma}$ preserves upward directed joins;

(c) $\left(f\left(g_{1}, \ldots, g_{n}\right)\right)^{\sigma}=f^{\sigma}\left(g_{1}^{\sigma}, \ldots g_{n}^{\sigma}\right)$.

The dual statements hold for dual operators.

We end this section with two fixpoint lemmas that will be crucial for the applications in Section 5. The first of these is a reformulation in terms of canonical extensions of Esakia's Lemma, first proved in [10]. (For a thorough treatment of Esakia's Lemma, and its connections to canonical extensions we refer the reader to [13].) 
Lemma 4.9. Let $\mathbf{L}$ be a lattice and let $f: L \rightarrow L$ be an order-preserving map. If $X \subseteq L$ is downward directed and closed under $f$, and $f$ is decreasing on $X$, then $f^{\sigma}(\bigwedge X)=\bigwedge X$ in $\mathbf{L}^{\sigma}$.

Proof. Let $y=\bigwedge X$ in $\mathbf{L}^{\sigma}$. Since $X$ is closed under $f$, we have $f(x) \geq y$ for each $x \in X$. Since $y \in K$, by Lemma 4.3(a), we have $f^{\sigma}(y)=\bigwedge\{f(a) \mid$ $a \in L, y \leq a\}$. By compactness, for each $a \in L$ with $a \geq y$, there exists a finite $X_{a} \subseteq X$ with $a \geq \bigwedge X_{a}$, and since $X$ is downward directed, there is an element $x_{a} \in X$ such that $a \geq x_{a}$. So for every $a \in L$ with $a \geq y$, we have $a \geq x_{a} \geq y$ for some $x_{a} \in X$. Hence $\bigwedge\{f(a) \mid a \in L, y \leq a\}=\bigwedge\{f(x) \mid$ $x \in X\}$. Now $X$ is closed under $f$, so $\bigwedge\{f(x) \mid x \in X\} \geq \bigwedge X$, but on the other hand, $f$ is decreasing on $X$, so $\bigwedge X \geq \bigwedge\{f(x) \mid x \in X\}$. Hence $f^{\sigma}(\bigwedge X)=\bigwedge X$, as claimed.

Lemma 4.10. Let $\mathbf{A}$ be a lattice-ordered algebra and $t(x, \bar{u})$ a term that is $\mathbf{A}$-isotone and $\mathbf{A}$-expanding. Let $p(x)=t(x, \bar{a})$ for some $\bar{a} \in A$. If $X \subseteq A$ is downward directed and closed under $p^{\mathbf{A}}$, and $p^{\mathbf{A}}$ is decreasing on $X$, then $t^{\mathbf{A}^{\sigma}}(\bigwedge X, \bar{a})=\bigwedge X$ in $\mathbf{A}^{\sigma}$.

Proof. Clearly $p: A \rightarrow A$ is an isotone map, so by Lemma 4.9, we have $\left(t^{\mathbf{A}}\right)^{\sigma}(\bigwedge X, \bar{a})=\left(p^{\mathbf{A}}\right)^{\sigma}(\bigwedge X)=\bigwedge X$. Since $t$ is expanding, $t^{\mathbf{A}^{\sigma}}(\bigwedge X, \bar{a}) \geq$ $\bigwedge X$. We also have $t^{\mathbf{A}^{\sigma}}(x, \bar{a})=t^{\mathbf{A}}(x, \bar{a})$ for every $x \in X$, as $X \cup\{\bar{a}\} \subseteq A$. Since $X$ is closed under $p^{\mathbf{A}}$, we get that $t^{\mathbf{A}^{\sigma}}(x, \bar{a}) \leq x$ holds for all $x \in X$, and hence $t^{\mathbf{A}^{\sigma}}(\bigwedge X, \bar{a}) \leq \bigwedge X$, as $t$ is isotone. Therefore, $t^{\mathbf{A}^{\sigma}}(\bigwedge X, \bar{a})=\bigwedge X$ as claimed.

\section{Case Studies}

In this section, we use Theorem 3.1 to establish the failure of coherence, and hence also uniform deductive interpolation, for various varieties of latticeordered algebras that are closed under canonical extensions. These case studies are all corollaries of the following result.

Theorem 5.1. Let $\mathcal{V}$ be a coherent variety of lattice-ordered algebras that is closed under canonical extensions and let $t(x, \bar{u})$ be any $\mathcal{V}$-expanding term that is order-preserving and decreasing in $\mathcal{V}$ with respect to $x$. Then $t$ is $n$-potent (with respect to $x$ ) in $\mathcal{V}$ for some $n \in \mathbb{N}$.

Proof. Let $t(x, \bar{u})$ be any $\mathcal{V}$-expanding term that is order-preserving and decreasing in $\mathcal{V}$ with respect to $x$. By Theorem 3.1, it suffices to show that 
$\mathcal{V}$ satisfies the fixpoint embedding condition $(\mathrm{FE})$ with respect to $t$. Let $\mathbf{A}$ be any algebra in $\mathcal{V}$ and let $a, \bar{b} \in A$. Then $\mathbf{A}$ embeds into its canonical extension $\mathbf{A}^{\sigma}$, and, since $\mathcal{V}$ is closed under canonical extensions, $\mathbf{A}^{\sigma} \in \mathcal{V}$. We identify $\mathbf{A}$ with its isomorphic copy in $\mathbf{A}^{\sigma}$, and let $X=\left\{t^{k}(a, \bar{b}) \mid k \in \mathbb{N}\right\}$. Since $\mathbf{A}^{\sigma}$ is complete, $\bigwedge X$ exists in $\mathbf{A}^{\sigma} .^{2}$ By assumption, $t$ is $\mathbf{A}$-expanding and $\mathbf{A}$-isotone, so Lemma 4.10 applies, yielding $t^{\mathbf{A}^{\sigma}}(\bigwedge X, \bar{b})=\bigwedge X$. This shows that (FE) holds, as required.

Since closure under canonical extensions will be essential for all our case studies, let us also note that to establish this property for a variety, it suffices to check that it holds for some generating class closed under ultraproducts.

Lemma 5.2. Let $\mathcal{V}$ be a variety of lattice-ordered algebras generated by a class $\mathcal{C}$ that is closed under ultraproducts. If $\mathcal{C}$ is closed under canonical extensions, then $\mathcal{V}$ is closed under canonical extensions .

Proof. Suppose that $\mathcal{C}$ is closed under ultraproducts and generates $\mathcal{V}$, i.e., $\mathcal{V}=\mathbb{H} \mathbb{S P}(\mathcal{C})$. Any direct product can be represented as a Boolean product of ultraproducts, so we have $\mathcal{V}=\mathbb{H S P}_{B} \mathbb{P}_{U}(\mathcal{C})=\mathbb{H S P}_{B}(\mathcal{C})$. By Lemma 4.4, canonical extensions commute with homomorphic images, subalgebras and Boolean products, so $\mathcal{C}^{\sigma} \subseteq \mathcal{C}$ implies $\mathcal{V}^{\sigma} \subseteq \mathcal{V}$

\subsection{Varieties of Boolean algebras with operators}

A Boolean algebra with operators is an algebra $\mathbf{A}=\langle A, \wedge, \vee, \neg, 0,1, \mathcal{O}\rangle$ such that $\langle A, \wedge, \vee, \neg, 0,1\rangle$ is a Boolean algebra, and $\mathcal{O}$ is a set of (multiplicative) operators. In this section, we will refer to $\mathcal{O}$ as the signature of $\mathbf{A}$, assuming tacitly that the Boolean operations are always present. We will also refer to operations in $\mathcal{O}$ simply as operators. (This is terminologically at odds with previous sections, but follows standard practice.)

Let $\operatorname{Clo}_{\text {ex }}(\mathcal{O})$ be the clone of all expanding terms over the signature $\mathcal{O}$. For $f \in \mathcal{O}$ of arity $n$, we define for each $k \leq n$, the unary operator $\square_{k}^{f} x=$ $f(\overline{0}, x, \overline{0}) \wedge x$, with $x$ occurring only at coordinate $k$; we define also $\square^{f} x=$ $\bigwedge_{0<k \leq n} \square_{k}^{f} x$. If $\mathcal{O}$ is finite, then we also let $\square x=\bigwedge_{f \in \mathcal{O}} \square^{f} x$. All these terms interpret to operators, so they belong to $\mathrm{Clo}_{\mathrm{ex}}(\mathcal{O})$ by Lemma 4.8 .

\footnotetext{
${ }^{2}$ Note that $\Lambda X$ may also exist in $\mathbf{A}$, and then $\bigwedge^{\mathbf{A}^{\sigma}} X \geq \bigwedge^{\mathbf{A}} X$. In general, $t\left(\bigwedge^{\mathbf{A}^{\sigma}} X\right)=\bigwedge^{\mathbf{A}^{\sigma}} X$ does not imply $t\left(\bigwedge^{\mathbf{A}} X\right)=\bigwedge^{\mathbf{A}} X$, so the required fixpoint for $t$ may always "escape" to the canonical extension.
} 
It is known that a variety $\mathcal{V}$ of Boolean algebras with operators of finite signature $\mathcal{O}$ admits equationally definable principal congruences (EDPC) if and only if $\square$ is $n$-potent for some $n \in \mathbb{N}$ (see, e.g., [27]). We will now show that for any variety $\mathcal{V}$ of Boolean algebras with operators, coherence implies EDPC for all varieties inheriting finitely many operators from $\mathcal{V}$, even if $\mathcal{V}$ itself is of infinite signature.

Theorem 5.3. Let $\mathcal{V}$ be a variety of Boolean algebras with operators of signature $\mathcal{O}$ that is closed under canonical extensions. Let $\mathcal{O}^{\prime}$ be a finite subset of $\mathrm{Clo}_{\mathrm{ex}}(\mathcal{O})$, and let $\mathcal{V}^{\prime}$ be the variety generated by term-reducts of members of $\mathcal{V}$ in the signature $\mathcal{O}^{\prime}$. If $\mathcal{V}$ is coherent, then $\mathcal{V}^{\prime}$ has the EDPC.

Proof. Let $\mathcal{C}=\left\{\mathbf{A}^{\sigma} \mid \mathbf{A} \in \mathcal{V}\right\}$, so that $\mathcal{V}=\mathbb{I S}(\mathcal{C})$. Defining $\mathcal{C}^{\prime}$ to be the class of $\mathcal{O}^{\prime}$-reducts of $\mathcal{C}$, we have $\mathcal{V}^{\prime}=\mathbb{H} \mathbb{S}\left(\mathcal{C}^{\prime}\right)$. The class $\mathcal{C}$ is closed under canonical extensions (because $\mathcal{V}$ is), and hence so is $\mathcal{C}^{\prime}$. Canonical extensions commute with subalgebras and homomorphic images (see Lemma 4.4), so $\mathcal{V}^{\prime}$ is also closed under canonical extensions. The result then follows by applying Theorem 5.1 with the term operation $\square$ defined for the signature $\mathcal{O}^{\prime}$.

A term $t$ is called positive if every occurrence of a variable lies within the scope of an even number of occurrences of $\neg$. Positive terms are expanding (cf. [24]), so Theorem 5.3 applies to any variety $\mathcal{V}$ of Boolean algebras with operators defined by identities containing positive terms. In particular, it applies to varieties with conjugate operators, or more broadly, to all varieties of finite signature, such that $\square$ has a term-definable conjugate, that is, an operator $g$ satisfying

$$
\bullet x \wedge y=0 \Longleftrightarrow x \wedge g(y)=0
$$

Such varieties are called cyclic. For cyclic varieties, the EDPC is equivalent to being a discriminator variety (see [27], or [26] for the special case of tense algebras), so a stronger result can be stated.

Corollary 5.4. Let $\mathcal{V}$ be a cyclic variety of Boolean algebras with operators that is closed under canonical extensions. If $\mathcal{V}$ is coherent, then $\mathcal{V}$ is a discriminator variety.

Theorem 5.3 applies of course to varieties of modal algebras. Let $\mathcal{K}$ be the variety of all modal algebras, and call any subvariety of $\mathcal{K}$ satisfying the equation $\square^{n+1} x \approx \square^{n} x$ for some $n \in \mathbb{N}$ weakly-transitive. 
Corollary 5.5. Let $\mathcal{V}$ be any subvariety of $\mathcal{K}$ that is closed under canonical extensions and not weakly transitive. Then $\mathcal{V}$ is not coherent and does not admit uniform deductive interpolation, and its first-order theory does not have a model completion.

In particular, neither of the varieties corresponding to the well-known modal logics $\mathrm{K}$ and $\mathrm{KT}$ are coherent, admit uniform deductive interpolation, or have a first-order theory that has a model completion. Note however, that there exist subvarieties of $\mathcal{K}$, such as the varieties corresponding to $\mathrm{K} 4$ and S4, that are both closed under canonical extensions and weakly transitive, but are still not coherent (see $[3,16,20]$ ). Failures of coherence, and therefore uniform (deductive) interpolation, for these and other non-transitive varieties of modal algebras have been established using our criterion (Theorem 3.1) in [28]. However, in this case, a unary term will not suffice and we make use of the ternary term $t(x, y, z)=\diamond(y \wedge \diamond(z \wedge x)) \wedge x$.

\subsection{Varieties of double-Heyting algebras}

A double-Heyting algebra is an algebra $\mathbf{A}=\langle A, \wedge, \vee, \rightarrow,-, 0,1\rangle$ such that $\langle A, \wedge, \vee, \rightarrow, 0,1\rangle$ is a Heyting algebra and $\langle A, \vee, \wedge,-, 0,1\rangle$ is a dual Heyting algebra, with - dually residuating $\vee$. We consider in this case the unary term $d(x)=(1-x) \rightarrow 0$. This term is decreasing in any double-Heyting algebra $\mathbf{A}$, and $d(\bigwedge X)=\bigwedge\{d(x) \mid x \in X\}$ holds for any $X \subseteq A$ for which $\bigwedge X$ is defined, so it also preserves meets of powers.

Lemma 5.6. The variety of double-Heyting algebras is closed under canonical extensions.

Proof. Let $\mathbf{A}=\langle A, \vee, \wedge, \rightarrow,-, 0,1\rangle$ be a double-Heyting algebra, and let $\mathbf{A}^{\sigma}=\left\langle A^{\sigma}, \vee^{\sigma}, \wedge^{\sigma}, \rightarrow^{\pi},-^{\sigma}, 0,1\right\rangle$. Then $\left\langle A^{\sigma}, \vee^{\sigma}, \wedge^{\sigma}, 0,1\right\rangle$ is a complete bounded distributive lattice. It is well known that $\rightarrow^{\pi}$ residuates $\wedge^{\sigma}$, so we only need to show that $-{ }^{\sigma}$ dually residuates $\vee^{\sigma}$. This follows by duality and Lemma 4.6, using the observation that - is an operator when viewed as a map from $A \times A^{\partial}$ to $A$.

It was shown in [40] that a variety of double-Heyting algebras has the EDPC if and only if it is a discriminator variety, and that this situation occurs if and only if the term $d$ defined above is $n$-potent for some $n \in \mathbb{N}$. Hence, we obtain the following analogue of Corollary 5.4. 
Theorem 5.7. Let $\mathcal{V}$ be a variety of double-Heyting algebras that is closed under canonical extensions. If $\mathcal{V}$ is coherent, then $\mathcal{V}$ is a discriminator variety.

Proof. Let $\mathcal{V}$ be a coherent variety of double-Heyting algebras that is closed under canonical extensions. By the observations at the beginning of this subsection, $\mathcal{V}$ satisfies the assumptions of Theorem 5.1 with the term $d$. Hence $\mathcal{V} \models d^{n+1}(x) \approx d^{n}(x)$ for some $n \in \mathbb{N}$, and the claim follows.

Since the variety of all double-Heyting algebras is not a discriminator variety, Lemma 5.6 combined with Theorem 5.7 yields the following result.

Corollary 5.8. The variety of double-Heyting algebras is not coherent and does not admit uniform deductive interpolation, and its first-order theory does not have a model completion.

This result may be somewhat surprising in view of the fact that Heyting algebras admit uniform deductive interpolation (cf. [21]), and bi-intuitionistic logic has the Craig interpolation property (cf. [29]).

\subsection{Varieties of residuated lattices}

A residuated lattice is an algebra $\mathbf{A}=\langle A, \wedge, \vee, \cdot, \backslash, /, \mathrm{e}\rangle$ such that $\langle A, \wedge, \vee\rangle$ is a lattice, $\langle A, \cdot, \mathrm{e}\rangle$ is a monoid, and for all $a, b, c \in A$,

$$
b \leq a \backslash c \Longleftrightarrow a \cdot b \leq c \Longleftrightarrow a \leq c / b .
$$

Residuated lattices expanded by a constant 0 are known as FL-algebras. To present results about FL-algebras and residuated lattices in a uniform way, we will view residuated lattices as FL-algebras satisfying the identity e $\approx 0$. We refer to [12] for further details regarding these structures and their role as algebraic semantics for substructural logics.

As remarked already in Section 4, the canonical extension of a residuated lattice must mix $\sigma$ and $\pi$ extensions of the basic operations. Hence, for a residuated lattice $\mathbf{A}$, its canonical extension is defined to be the algebra $\mathbf{A}^{\sigma}=\left\langle A^{\sigma}, \vee^{\sigma}, \wedge^{\sigma}, \cdot^{\sigma}, \backslash^{\pi}, /^{\pi}, \mathrm{e}^{\sigma}\right\rangle$. Note that the multiplication operation is an operator, and the divisions (with appropriately dualised coordinates) are dual operators. When so defined, $\mathbf{A}^{\sigma}$ is a residuated lattice, showing that the variety of residuated lattices is closed under canonical extensions. Many

other important varieties of residuated lattices are also closed under canonical extensions as illustrated by the following two lemmas. 
Lemma 5.9. Let $\mathcal{V}$ be a variety of FL-algebras defined (relative to the variety of all FL-algebras) by any combination of the following identities:

- $\mathrm{e} \approx 0$ (residuated lattices);

- $x \leq \mathrm{e}$ (integral FL-algebras, or $\mathrm{FL}_{o}$-algebras);

- $0 \leq x$ (zero-bounded FL-algebras, or $\mathrm{FL}_{i}$-algebras);

- $x y \approx y x$ (commutative FL-algebras, or $\mathrm{FL}_{e}$-algebras);

- $x \leq x^{2}$ (square-increasing FL-algebras, or $\mathrm{FL}_{c}$-algebras);

- $0 /(x \backslash 0) \approx(0 / x) \backslash 0$ (cyclic FL-algebras);

- $0 /(x \backslash 0) \approx x \approx(0 / x) \backslash 0$ (involutive FL-algebras);

- $(\mathrm{e} \wedge x)^{k} y \approx y(\mathrm{e} \wedge x)^{k}$, for some $k \in \mathbb{N}$ (Hamiltonian FL-algebras);

- $x \wedge(y \vee z) \approx(x \wedge y) \vee(x \wedge z)$ (distributive FL-algebras).

Then $\mathcal{V}$ is closed under canonical extensions.

Proof. All these claims are corollaries of the results in Chapter 6 of [12], but to give the reader an idea of how the proofs proceed, we give a proof for the Hamiltonian case here. Suppose that $\mathbf{A} \models(\mathrm{e} \wedge x)^{k} y \approx y(\mathrm{e} \wedge x)^{k}$ for some $k \in \mathbb{N}$. We will show that this identity also holds in $\mathbf{A}^{\sigma}$.

Since multiplication is an operator and $\wedge$ is order-preserving, Lemma 4.8 applies (recursively) to the terms $(\mathrm{e} \wedge x)^{k} y$ and $y(\mathrm{e} \wedge x)^{k}$. This yields

$$
\begin{aligned}
& \left.\left(\left(\mathrm{e} \wedge^{\sigma} x\right)^{\sigma}\right)^{k} \cdot{ }^{\sigma} y=\left((\mathrm{e} \wedge x)^{k}\right)^{\sigma} \cdot{ }^{\sigma} y\right) \\
& =\left((\mathrm{e} \wedge x)^{k} y\right)^{\sigma} \\
& =\left(y(\mathrm{e} \wedge x)^{k}\right)^{\sigma} \\
& =y \cdot{ }^{\sigma}\left(\left(\mathrm{e} \wedge^{\sigma} x\right)^{\sigma}\right)^{k},
\end{aligned}
$$

showing that $\mathbf{A}^{\sigma} \models(\mathrm{e} \wedge x)^{k} y \approx y(\mathrm{e} \wedge x)^{k}$ as required.

Lemma 5.10. Let $\mathcal{V}$ be a variety of FL-algebras that is closed under canonical extensions. Then also the variety $\mathcal{V}^{\ell}$ of semilinear algebras generated by the linearly ordered members of $\mathcal{V}$ is closed under canonical extensions.

Proof. This result follows from Theorem 6.8 in [14], but let us sketch a direct proof. Let $\mathcal{V}$ be a variety of FL-algebras that is closed under canonical extensions, and let $\mathcal{C}$ be the class of chains (linearly ordered members) of $\mathcal{V}$. Ultraproducts of chains are chains, and canonical extensions of chains are chains, so the claim follows by Lemma 5.2. 
It was shown in [11] that a Hamiltonian variety of residuated lattices $\mathcal{V}$ has the EDPC if and only if $\mathcal{V} \models(\mathrm{e} \wedge x)^{n+1} \approx(\mathrm{e} \wedge x)^{n}$ for some $n \in \mathbb{N}$. Let $t(x)=(\mathrm{e} \wedge x)^{2}$. Since $t$ is decreasing and expanding in all residuated lattices, an application of Theorem 5.1 yields the following result.

Theorem 5.11. Let $\mathcal{V}=\mathbb{I S P}(\mathcal{C})$ be a coherent variety of residuated lattices such that $\mathcal{C}$ is closed under canonical extensions. Then $\mathcal{V} \models(\mathrm{e} \wedge x)^{n+1} \approx$ $(\mathrm{e} \wedge x)^{n}$ for some $n \in \mathbb{N}$ and if $\mathcal{V}$ is also Hamiltonian, then $\mathcal{V}$ has the EDPC.

Corollary 5.12. Let $\mathcal{V}=\mathbb{I S P}(\mathcal{C})$ be any variety of residuated lattices such that $\mathcal{C}$ is closed under canonical extensions and $\mathcal{V} \not(\mathrm{e} \wedge x)^{n+1} \approx(\mathrm{e} \wedge x)^{n}$ for all $n \in \mathbb{N}$. Then $\mathcal{V}$ is not coherent and does not admit uniform deductive interpolation, and its first-order theory does not have a model completion.

The lattice-dual form of Theorem 5.11 also holds. That is, if $\mathcal{V}$ satisfies the assumptions of the theorem, then $\mathcal{V}=(\mathrm{e} \vee x)^{m+1} \approx(\mathrm{e} \vee x)^{m}$ for some $m \in \mathbb{N}$. Of the varieties mentioned in Lemma 5.9 , those that satisfy both $(\mathrm{e} \wedge x)^{n+1} \approx$ $(\mathrm{e} \wedge x)^{n}$ for some $n \in \mathbb{N}$, and $(\mathrm{e} \vee x)^{m+1} \approx(\mathrm{e} \vee x)^{m}$ for some $m \in \mathbb{N}$, are term-equivalent to Heyting algebras. Hence, we obtain failures of coherence, uniform deductive interpolation, and existence of a model completion for the first-order theory for varieties of residuated lattices corresponding to all 'fundamental' substructural logics, including $F L, F L_{c}, F L_{e}, F_{w}$, and $F_{e w}$, and their involutive versions, including MALL, the fragment of Linear Logic without exponentials.

\subsection{Varieties of lattices}

Our final negative result concerns the variety $\mathcal{L}$ at of lattices. Here we remove the assumption that the signature contains at least one constant. Let $\mathcal{L}$ be an arbitrary algebraic signature, and let $\mathcal{V}$ be a variety of $\mathcal{L}$-algebras. The presence or absence of a constant does not affect the definition of coherence, but Proposition 2.1 and hence Theorem 2.3 are not quite correct in this more general setting. Rather than reformulating these results in their entirety, let us just extract the one result that we need. From the proof of Theorem 2.3, we obtain directly that the following are equivalent:

(1) $\mathcal{V}$ is coherent.

(2) For any finite sets $\bar{x}, \bar{y}$ such that $\mathbf{F}(\bar{y})$ exists and any compact congruence $\Theta$ on $\mathbf{F}(\bar{x}, \bar{y})$, the congruence $\Theta \cap F(\bar{y})^{2}$ on $\mathbf{F}(\bar{y})$ is compact. 

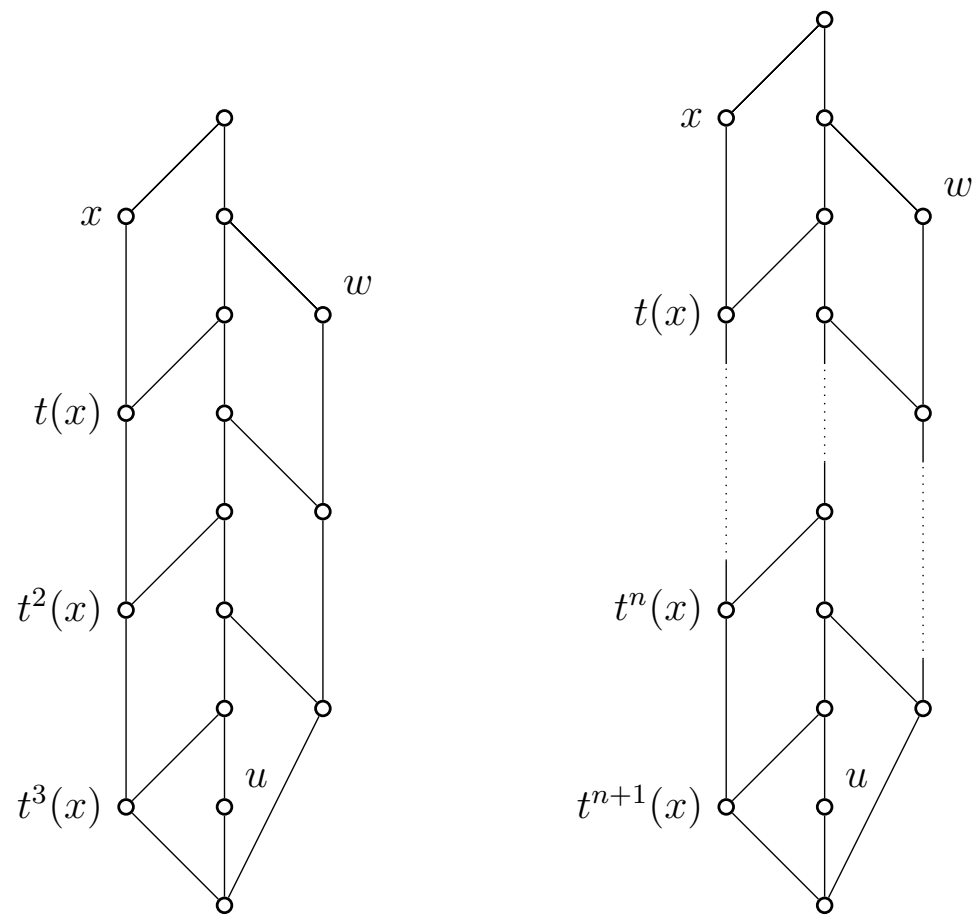

Figure 1: Lattices falsifying $t^{n}(x, u, w) \approx t^{n+1}(x, u, w)$ for $n \in \mathbb{N}$. Left $\mathbf{H}_{2}$; right $\mathbf{H}_{n}$.

Lemma 5.13. Let $\mathcal{W}$ be a coherent variety of lattices closed under canonical extensions, and let $s(x, \bar{u})$ be any lattice term. Then the term $s \wedge x$ is $n$-potent in $\mathcal{W}$ for some $n \in \mathbb{N}$.

Proof. Since $s \wedge x$ is a composition of lattice terms, by Lemma 4.5, it is $\mathcal{W}$-expanding. The claim then follows immediately from Theorem 5.1.

Let us consider now the ternary lattice term

$$
t(x, u, w)=(u \vee(w \wedge(u \vee x))) \wedge x .
$$

For each $n \in \mathbb{N}$, let $\mathbf{H}_{n}$ be the lattice with $8+4 n$ elements depicted in Figure 1 with an assignment to the variables $x, u, w$. (Readers familiar with lattice theory may recognise $\mathbf{H}_{n}$ as a finite version of the herringbone of [2].) By considering this Hasse diagram and assignment, it is clear that $\mathbf{H}_{n} \forall$ $t(x, u, w) \leq t^{n+1}(x, u, w)$. 
Corollary 5.14. Let $\mathcal{V}$ be a variety of lattices closed under canonical extensions and such that $\mathbf{H}_{n} \in \mathcal{V}$ for all $n \in \mathbb{N}$. Then $\mathcal{V}$ is not coherent and does not admit deductive uniform interpolation, and its first-order theory does not have a model completion.

Since the variety $\mathcal{L}$ at of all lattices is obviously closed under canonical extensions, we obtain the following negative result, first proved explicitly by Schmidt in [38]. ${ }^{3}$

Corollary 5.15. The variety $\mathcal{L}$ at of all lattices is not coherent and does not admit deductive uniform interpolation, and its first-order theory does not have a model completion.

Corollary 5.14 can also be used to establish failures of coherence for other varieties of lattices. Recall that a lattice $\mathbf{L}$ is said to be of width $k$ if the longest antichain in $\mathbf{L}$ has $k$ elements. Let $\mathcal{L W}_{n}$ be the variety of lattices generated by all lattices of width at most $n$.

Lemma 5.16. For each $n \in \mathbb{N}$, the variety $\mathcal{L W}_{n}$ is closed under canonical extensions.

Proof. Let $\mathcal{W}_{n}$ be the class of lattices of width at most $n$. Since the property of having width at most $n$ is definable by a first-order sentence, $\mathcal{W}_{n}$ is closed under ultraproducts. Moreover, it is not difficult to show that the canonical extension of a lattice of width $k$ is itself of width $k$, so $\mathcal{W}_{n}$ is closed under canonical extensions. The claim then follows by Lemma 5.2.

The variety $\mathcal{L W}_{1}$, generated by chains, is precisely the variety of distributive lattices. Also, it is proved in [34] that $\mathcal{L W}_{2}$ is generated by $\mathbf{N}_{5}$ (the pentagon). So $\mathcal{L W}_{1}$ and $\mathcal{L W}_{2}$ are locally finite and hence coherent. Since each $\mathbf{H}_{n}(n \in \mathbb{N})$ is of width 3, Corollary 5.14 yields the following result.

Corollary 5.17. The variety $\mathcal{L W}_{n}$ is coherent if and only if $n \in\{1,2\}$. Moreover, for any $n \geq 3$, the variety $\mathcal{L} \mathcal{W}_{n}$ does not admit deductive uniform interpolation, and its first-order theory does not have a model completion.

\footnotetext{
${ }^{3}$ It is noted in [38], however, that an unpublished example exhibiting the failure of coherence had already been given by R. McKenzie.
} 


\section{References}

[1] M. Alizadeh, F. Derakhshan, and H. Ono, Uniform interpolation in substructural logics, Rev. Symb. Log. 7 (2014), no. 3, 455-483.

[2] H. Bauer and W. Poguntke, Lattices of width three: an example and a theorem, Contributions to general algebra (Proc. Klagenfurt Conf., Klagenfurt, 1978), Heyn, Klagenfurt, 1979, pp. 47-54.

[3] M. Bílková, Uniform interpolation and propositional quantifiers in modal logics, Studia Logica 85 (2007), 1-31.

[4] K.G. Choo, K.Y. Lam, and E. Luft, On free product of rings and the coherence property, Algebraic K-theory, II: "Classical" algebraic K-theory and connections with arithmetic (Proc. Conf., Battelle Memorial Inst., Seattle, Wash., 1972), Springer, 1973, pp. 135-143. Lecture Notes in Math., Vol. 342.

[5] J. Czelakowski, Sentential logics and Maehara interpolation property, Studia Logica 44 (1985), no. 3, 265-283.

[6] _ Fregean logics and the strong amalgamation property, Bull. Sect. Logic $\mathbf{2 6}$ (2007), no. 3/4, 105-116.

[7] G. D'Agostino and M. Hollenberg, Logical questions concerning the $\mu$-calculus, J. Symb. Log. 65 (2000), no. 1, 310-332.

[8] G. D'Agostino, Uniform interpolation, bisimulation quantifiers, and fixed points, Proc. TbiLLC'05, 2005, pp. 96-116.

[9] P. Eklof and G. Sabbagh, Model-completions and modules, Ann. Math. Logic 2 (1970/1971), no. 3, 251-295.

[10] L.L. Esakia, Topological Kripke models, Dokl. Akad. Nauk SSSR 214 (1974), 298-301.

[11] N. Galatos, Varieties of residuated lattices, PhD thesis, Vanderbilt University, Nashville, Tennessee, 2003.

[12] N. Galatos, P. Jipsen, T. Kowalski, and H. Ono, Residuated Lattices: An Algebraic Glimpse at Substructural Logics, Studies in Logic and the Foundations of Mathematics, Elsevier, 2007.

[13] M. Gehrke, Canonical extensions, Esakia spaces, and universal models, Leo Esakia on duality in modal and intuitionistic logics, Outst. Contrib. Log., vol. 4, Springer, 2014, pp. 9-41.

[14] M. Gehrke and J. Harding, Bounded lattice expansions, J. Algebra 238 (2001), no. 1, $345-371$.

[15] S. Ghilardi, An algebraic theory of normal forms, Ann. Pure Appl. Logic 71 (1995), no. $3,189-245$.

[16] S. Ghilardi and M. Zawadowski, Sheaves, Games, and Model Completions: A Categorical Approach to Nonclassical Propositional Logics, Springer, Dordrecht, 2002. 
[17] van Gool. S., G. Metcalfe, and C. Tsinakis, Uniform interpolation and compact congruences, Ann. Pure Appl. Logic 168 (2017), 1827-1948.

[18] V. Gould, Coherent monoids, J. Austral. Math. Soc. Ser. A 53 (1992), no. 2, 166-182.

[19] S. Ghilardi, C. Lutz, and F. Wolter, Did I damage my ontology? A case for conservative extensions in description logics, Proc. KR'06, AAAI Press, 2006, pp. 187-197.

[20] S. Ghilardi and M. Zawadowski, Undefinability of propositional quantifiers in the modal system S4, Studia Logica 55 (1995), no. 2, 259-271.

[21] _ Model completions and r-Heyting categories, Ann. Pure Appl. Logic $\mathbf{8 8}$ (1997), no. 1, 27-46.

[22] G. Higman, Subgroups of finitely presented groups, Proc. Roy. Soc. Ser. A 262 (1961), $455-475$.

[23] B. Jónsson, Extensions of relational structures, Proc. International Symposium on the Theory of Models, Berkeley, 1965, pp. 146-157.

[24] _ On the canonicity of Sahlqvist identities, Studia Logica 53 (1994), 473-491.

[25] O. Kharlampovich and M. Sapir, Algorithmic problems in varieties, a survey, Internat. J. Algebra Comput. 12 (1995), 379-602.

[26] T. Kowalski, Varieties of tense algebras, Rep. Math. Logic 32 (1998), 53-95.

[27] T. Kowalski and M. Kracht, Semisimple varieties of modal algebras, Studia Logica 83 (2006), no. 1-3, 351-363.

[28] T. Kowalski and G. Metcalfe, Coherence in modal logic, Proc. AiML'18, College Publications, 2018, pp. 236-251.

[29] T. Kowalski and H. Ono, Analytic cut and interpolation for bi-intuitionistic logic, Rev. Symb. Log. 10 (2017), no. 2, 259-283.

[30] C. Lutz and F. Wolter, Foundations for uniform interpolation and forgetting in expressive description logics, Proc. IJCAI'11, 2011, pp. 989-995.

[31] L.L. Maksimova, Craig's theorem in superintuitionistic logics and amalgamable varieties of pseudo-Boolean algebras, Algebra Logika 16 (1977), 643-681.

[32] G. Metcalfe, F. Montagna, and C. Tsinakis, Amalgamation and interpolation in ordered algebras, J. Algebra 402 (2014), 21-82.

[33] J. Marti, F. Seifan, and Y. Venema, Uniform interpolation for coalgebraic fixpoint logic, CALCO, LIPIcs, vol. 35, Schloss Dagstuhl - Leibniz-Zentrum für Informatik, 2015, pp. 238-252.

[34] O.T. Nelson, Subdirect decompositions of lattices of width two, Pacific J. Math. 24 (1968), no. 3, 519-523.

[35] H. Ono, Interpolation and the Robinson property for logics not closed under the Boolean operations, Algebra Universalis 23 (1986), 111-122. 
[36] D. Pigozzi, Amalgamation, congruence-extension, and interpolation properties in algebras, Algebra Universalis 1 (1972), 269-349.

[37] A.M. Pitts, On an interpretation of second-order quantification in first-order intuitionistic propositional logic, J. Symbolic Logic 57 (1992), 33-52.

[38] P.H. Schmidt, Algebraically complete lattices, Algebra Universalis 17 (1983), 135-142.

[39] V.Yu. Shavrukov, Subalgebras of diagonalizable algebras of theories containing arithmetic, Dissertationes Mathematicae, vol. 323, Polska Akademia Nauk., Instytut Matematyczny, Warsaw, 1993.

[40] C.J. Taylor, Discriminator varieties of double-Heyting algebras, Rep. Math. Logic 51 (2016), 3-14.

[41] W. Taylor, Uniformity of congruences, Algebra Universalis 4 (1974), 342-360.

[42] A. Visser, Uniform interpolation and layered bisimulation, Gödel '96: Logical foundations on mathematics, computer science and physics - Kurt Gödel's legacy (P. Hájek, ed.), Springer Verlag, 1996.

[43] W.H. Wheeler, Model-companions and definability in existentially complete structures, Israel J. Math. 25 (1976), no. 3, 305-330.

[44] _ A characterization of companionable, universal theories, J. Symbolic Logic 43 (1978), no. 3, 402-429.

[45] A. Wroński, On a form of equational interpolation property, Foundations of Logic and Linguistics: Problems and their Solutions (G. Dorn and P. Weingartner, eds.), Springer, Boston, 1985, pp. 23-29. 